Research Paper

\title{
Dual-function of Baicalin in nsPEFs-treated Hepatocytes and Hepatocellular Carcinoma cells for Different Death Pathway and Mitochondrial Response
}

\author{
Yubo Wang ${ }^{1 *}$, Shengyong Yin ${ }^{*}$, Yuan Zhou ${ }^{*}$, Wuhua Zhou ${ }^{1,2}$, Tianchi Chen ${ }^{1}$, Qinchuan Wu1 ${ }^{1}$, Lin Zhou ${ }^{\circledR}$, \\ Shusen Zheng ${ }^{1 凶}$ \\ 1. Department of Hepatobiliary and Pancreatic Surgery, First Affiliated Hospital, Key Laboratory of Combined Multi-Organ Transplantation, Ministry of \\ Public Health, School of Medicine, Zhejiang University, Hangzhou, Zhejiang Province 310003, China \\ 2. Department of hepatobiliary and pancreatic surgery, Taihe Hospital, Hubei University of Medicine, Hubei, China \\ *Contributed equally.
}

$\triangle$ Corresponding authors: Shusen Zheng, Division of Hepatobiliary and Pancreatic Surgery, Department of Surgery, First Affiliated Hospital, School of Medicine, Zhejiang University, No. 79 Qingchun Road, Hangzhou 310003, China. Tel: +86 571 87236466; Email: shusenzheng@zju.edu.cn. Lin Zhou, Division of Hepatobiliary and Pancreatic Surgery, Department of Surgery, First Affiliated Hospital, School of Medicine, Zhejiang University, No. 79 Qingchun Road, Hangzhou 310003, China. Tel: +86 571 87236601; Email: zhoulin99@zju.edu.cn

(c) The author(s). This is an open access article distributed under the terms of the Creative Commons Attribution License (https://creativecommons.org/licenses/by/4.0/). See http://ivyspring.com/terms for full terms and conditions.

Received: 2019.03.13; Accepted: 2019.08.03; Published: 2019.09.07

\begin{abstract}
Nanosecond pulsed electric fields (nsPEFs) is emerged as a potential curative modality to ablate hepatocellular carcinoma (HCC). The application of local ablation is usually limited by insufficiency of liver function. While baicalin, a flavonoid isolated from Scutellaria baicalensis Georgi, has been proven to possess both anti-tumor and protective effects. Our study aimed to estimate different responses of hepatic cancer cells and hepatocytes to the combination of nsPEFs and baicalin. Cell viability, apoptosis and necrosis, mitochondrial transmembrane potential (MTP) and reactive oxygen species (ROS) were examined by CCK-8, FCM, JC-1 and fluorescent probe, respectively. After treatment by nsPEFs, most hepatocytes died by apoptosis, nevertheless, nearly all cancer cells were killed through necrosis. Low concentration of baicalin synergically enhanced nsPEFs-induced suppression and necrosis of $\mathrm{HCC}$ cells, nevertheless, the application of baicalin protected normal hepatocytes from the injury caused by nsPEFs, owing to elevating mitochondrial transmembrane potential and reducing ROS generation. Our work provided an advantageous therapy for HCC through the enhanced combination treatment of nsPEFs and baicalin, with which could improve the tumor-ablation effect and alleviate the injury of hepatic tissues simultaneously.
\end{abstract}

Key words: Dual Function, Hepatocellular Carcinoma, Nanosecond pulsed electric fields, Baicalin, Mitochondrial transmembrane potential

\section{Introduction}

Liver cancer, of which about $75 \%-85 \%$ cases are hepatocellular carcinoma (HCC), is quite prevalent and lethal worldwide [1]. Less than $30 \%$ of HCC patients have an opportunity to undergo surgery due to poor physical condition, major vascular invasion or shortage of organ supply. For most cases of HCC, local treatments, comprising trans arterial chemoembolization (TACE), radiofrequency ablation (RFA) and percutaneous ethanol injection (PEI), are widely adopted due to unavailable resection of tumor [2-4]. However, these local strategies are frequently limited by multiple complications, for instance, thermal and chemical injuries. To surmount these defects, a novel treatment nanosecond pulsed electric fields (nsPEFs), which employs nanosecond duration electrical pulses with utmost voltage and field strength, has been lately developed to ablate solid tumor by non-thermal way [3]. Instantaneous huge 
power of nsPEFs triggers death of tumor cells but is merely harmful to intrahepatic ducts [5]. NsPEFs can induce cell death through several mechanisms, mainly including the reversible electroporation of plasma membrane (PM) and mitochondria damage [6, 7]. These high intensity pulses expand the membrane permeability and ultimately permit small molecules to penetrate the plasma membrane such as calcium or dyes, for instance, propidium (PI) and trypan blue (TB) $[8,9]$. In addition, the latest evidence has shown that the application of nsPEFs with much shorter pulse duration has more impact on intracellular organelle than plasma membrane [10], which leads to the dissipation of mitochondria transmembrane potential [7]. Furthermore, nsPEFs can trigger calcium overload [11], stress responses [12], apoptosis [11, 13, 14] and diverse signal kinase pathways activation in cancer cells [15-17], and the ablation effect of nsPEFs has been validated on various malignancies including hepatocellular carcinoma [18], melanoma [19], pancreatic cancer [20], squamous cell carcinoma [21] etc.

Although nsPEFs can effectively ablate hepatic tumors, it is inevitable for nsPEFs to damage normal hepatic tissues, which might cause liver insufficiency. In order to improve the therapeutic effect of nsPEFs, baicalin, the major flavonoid and main active ingredient purified from traditional Chinese medicine Scutellaria baicalensis Georgi, whose chemical constitution is known [22], is employed. Baicalin has been reported as an effective agent exhibiting multiple pharmacological functions, for instance, anti-tumor, anti-inflammatory and anti-oxidation [23-25]. These pharmacological functions are depend on the arrest of cell cycle, induction of apoptosis, reduction of reactive oxygen species (ROS) and stabilization of mitochondrial transmembrane potential (MTP) [26, 27]. Baicalin or baicalein, $90 \%$ of which would convert into baicalin in blood, has been reported to be lethal to hepatic tumor by suppressing tumor migration and invasion, inducing apoptosis and inhibiting tumor growth [28, 29].

Since the anti-tumor function of nsPEFs has been validated, we hypothesized that the application of nsPEFs could effectively ablate HCC and the normal hepatic tissue damage within the range of effective electric field could be prevented by agents, such as baicalin. In this study, low concentration of baicalin was used after the application of nsPEFs to enhance the tumor-elimination capability and protect normal hepatocytes from the injury caused by nsPEFs simultaneously. The results demonstrated a dual function that the combined therapy could inhibit HCC cells more effectively by enhancing necrotic cell death but alleviate the damage of normal hepatocytes by preserving mitochondrial transmembrane potential and cleaning up cellular reactive oxygen species. These findings elicited a potential clinical strategy to eliminate hepatocellular carcinoma more sufficiently while alleviating the damage of normal hepatic tissues and provided a conceivable clinical guidance for nsPEFs.

\section{Materials and Methods}

\section{Cell culture}

Human normal hepatocyte line QSG-7701 and human hepatocellular carcinoma cell line MHCC-97H were purchased from the Chinese Academy of Science. High metastatic HCC cell line HCC-LM3 was purchased from the Liver Cancer Institute, Zhongshan Hospital, Fudan University. QSG-7701 cells were maintained in RPMI-1640 (Gibco-Invitrogen, Carlsbad, CA, USA) and MHCC-97H, HCC-LM3 cells were maintained in DMEM (Gibco-Invitrogen, Carlsbad, CA, USA), and both mediums were supplemented with $10 \%$ fetal bovine serum (FBS, SAFC Biosciences, Lenexa, KS, USA), 100 unit $/ \mathrm{ml}$ penicillin and $100 \mathrm{mg} / \mathrm{ml}$ streptomycin (SigmaAldrich, St. Louis, MO, USA).

\section{Isolation and culture of primary mouse hepatocytes}

The primary mouse hepatocytes were isolated from 28-day-old male C57BL/ 6 mice. The mouse was first anaesthetized and the liver was perfused with Krebs-Ringer buffer and collagenase IV (Sigma Aldrich, St. Louis, MO, USA) without calcium and magnesium. Fibroblasts and liver non-parenchymal cells were removed through DMEM elution. The primary mouse hepatocytes were seeded onto a collagen-coated plate and cultured with the special complete medium of primary mouse hepatocytes (Procell, Wuhan, China) (Figure 1D). All animal experiments were performed in accordance with protocols and regulations of the Experimental Animal Ethics Committee of the First Affiliated Hospital of Zhejiang University (Hangzhou, Zhejiang, China).

\section{NsPEFs generator and nsPEFs application}

NsPEFs generator's essential parameter adjustment was shown in our previous study [35] (Figure 1A). Waveforms were monitored with a digital phosphor oscilloscope (DPO4054, Tektronix, USA, Figure 1C) equipped with a high voltage probe (P6015A, Tektronix, USA). Cells were harvested with trypsin (Gibco-Invitrogen, Carlsbad, CA, USA) and re-suspended in advance preparing medium to a concentration of $2.0 \times 10^{6}$ cells $/ \mathrm{ml}$. Antibiotic free pulse mediums included RPMI-1640 containing 10\% FBS for QSG-7701 and DMEM containing 10\%FBS for 
MHCC-97H and HCC-LM3. $1 \mathrm{ml}$ of cell suspension was placed into a $0.4 \mathrm{~cm}$ gap cuvette (Biosmith, aluminum plate electrodes, Figure 1B) and exposed to 100ns, $1 \mathrm{HZ}, 30,40,50,60,80$ pulses at 15,25 and 40 $\mathrm{kV} / \mathrm{cm}$ electric field strength, respectively. These nsPEFs-treated cells (8000 cells/well) were seeded into 96-well plates and incubated for $24 \mathrm{~h}$, then their death/viability was detected by CCK-8 (Dojindo, Kumamoto, Japan) assay.

\section{Baicalin exposure and combination treatment of baicalin and nsPEFs}

For baicalin treatment, cells were seeded into 96-well plates and treated by baicalin (Solorbio, Beijing, China) with concentration of $0.1,1,10,20,40$, $80,160,320$ and $640 \mu \mathrm{M}$, respectively, for $24 \mathrm{~h}$ or $48 \mathrm{~h}$, and then their viability was measured with CCK-8 assay. For combined treatment of baicalin and nsPEFs, cells were first exposed to 40P, 15, 25 and $40 \mathrm{kv} / \mathrm{cm}$ nsPEFs, then placed into 96-well plates (8000/well). Cells were cultured for $6 \mathrm{~h}$ to adhere to the plate and then incubated with $0.625 \mu \mathrm{M}$ baicalin for $24 \mathrm{~h}$ or $48 \mathrm{~h}$, following by viability measurement with CCK-8 assay.
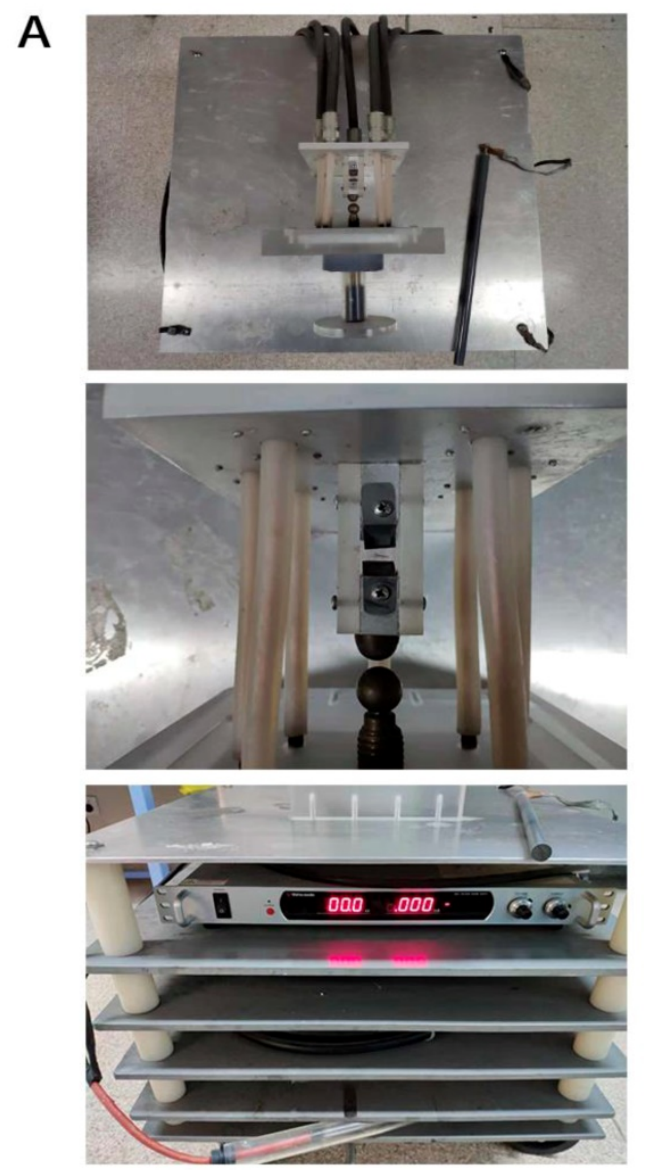

\section{Cell apoptosis and necrosis analysis}

Cell apoptosis and necrosis was quantitatively measured using Annexin V-FITC apoptosis detection kit (Dojindo, Kumamoto, Japan) by flow cytometry (FCM). Cells were harvested and washed by PBS, then dyed by FITC and PI $(8 \mu \mathrm{l} / \mathrm{ml})$ for $30 \mathrm{~min}$ in room temperature before detected by FCM. Double-negative FITC-/PI-, single positive PI+, single positive FITC+ and double-positive FITC+/PI+ represented the living cells, mechanical injury cells, early phase apoptotic cells and late phase apoptotic or necrotic cells, respectively.

\section{Western-blot assay}

RIPA buffer was utilized in lysing cells. All protein concentration was quantified by BCA method. $25 \mu \mathrm{g}$ of proteins from each group were loaded on ExpressPLUSTMPAGE gels (GenScript, USA) and then transferred on PVDF membranes before incubated with primary antibodies (1:2000) overnight. After incubation with HRP-conjugated secondary antibody (1:5000) for $2 \mathrm{~h}$, proteins were detected by EZ-ECL (Biological Industries, Israel). Anti-PAR (ab14459), anti-cleaved PARP-1 (ab32561) and anti- $\beta$-actin (ab8226) were purchased from Abcam (Cambridge, UK).
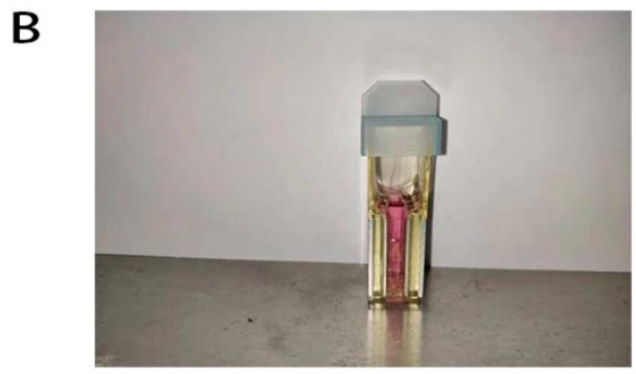

C

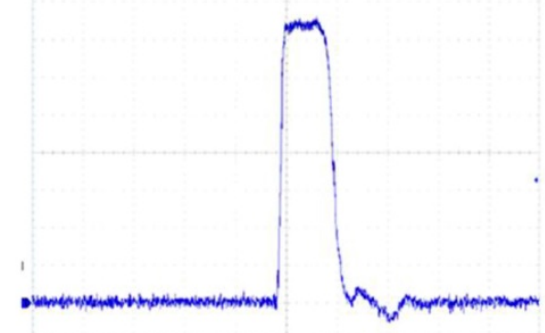

D

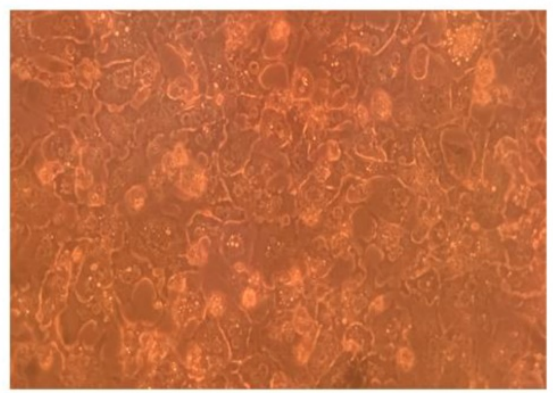

Figure 1: Demonstration of nsPEFs generator (A), cuvettes (B), basic wave of nsPEFs $(C)$ and primary mouse hepatocytes (D). 


\section{Mitochondrial transmembrane potential measurement}

The tetraethylbenzimidazolylcarbocyanine iodide (JC-1) is a cationic dye that accumulates in energized mitochondria. Cells were harvested and washed after handled by different treatment for $24 \mathrm{~h}$. For positive control, untreated cells were first mixed with $3 \mu \mathrm{l} / \mathrm{ml} \mathrm{CCCP}$ and PBS for $1 \mathrm{~h}$ in $37^{\circ} \mathrm{C}$ before mixed with $1 \mu \mathrm{l} / \mathrm{ml}$ JC-1 (MultiScience, Hangzhou, China) for $30 \mathrm{~min}$ in $37^{\circ} \mathrm{C}$. For other groups, $1 \mu \mathrm{l} / \mathrm{ml}$ JC-1 were mixed with cells and PBS for $30 \mathrm{~min}$ in $37^{\circ} \mathrm{C}$. Eventually, red fluorescence indicated by $\mathrm{PE}$ is detected when JC-1 accumulation in mitochondria is sustained by the normal cellular electrochemical potential gradient, or green fluorescence indicated by FITC is present when JC-1 is dispersed into cytoplasm for the dissipation of MTP through FCM.

\section{Intracellular ROS detection by cell ROS reagent}

NsPEFs-treated cells were incubated in the presence or absence of $0.625 \mu \mathrm{M}$ baicalin for $24 \mathrm{~h}$, and then washed twice with PBS before stained with $2 \mu \mathrm{l} / \mathrm{ml}$ CellROX Green Reagent (ThermoFisher, MA, U.S.A) for $30 \mathrm{~min}$ in $37^{\circ} \mathrm{C}$ and light-resistant incubator. Ultimately, the concentration of ROS was measured by FCM and fluorescent microscopy, during which the detectable bright green fluorescent signal (at the light wave length of $488 \mathrm{~nm}$, or Alexa Fluor 488-A) presented the amount of ROS.

\section{Cell Viability}

Cells were placed into 96-well plates and incubated with $10 \mu \mathrm{l} \mathrm{CCK}-8$ solution at $37^{\circ} \mathrm{C}$. Each sample was replicated 6 times. After 1 hour, the optical density was obtained at $450 \mathrm{~nm}$ by a spectrophotometer (ELx800; BioTek Instruments, Inc., Vermont, VT, U.S.A). The relative survival rate was calculated by the ratio of OD values of experiment group to OD values of control group.

\section{Statistical analysis}

Raw data were normalized by Microsoft Excel 2010 and figures were generated by GraphPad Prism 5.0 (GraphPad Software, San Diego, CA, U.S.A). Statistical analysis was performed with SPSS 16.0 for windows (SPSS, Chicago, IL, U.S.A). Quantitative variables were expressed as means \pm SD. For FCM data, FlowJo V10 (FlowJo LLC, Ashland, OR, U.S.A) was participated. Student's t-test, one-way ANOVA and $\chi 2$ analysis were performed to analyze variance. Results were considered statistically significant at $\mathrm{P}<$ 0.05 . All experiments were repeated three times.

\section{Results}

\section{Baicalin was more toxic to cancer cells while less toxic to hepatocytes}

Previous researches have proved that baicalin could suppress HCC cells including HepG2 and SMMC-7721 and has fewer side effects to normal hepatocytes, the Chang liver cell line [26, 28, 29]. In our study, we first assessed the toxicity of baicalin on HCC cell lines MHCC-97H and HCC-LM3, normal human hepatic cell line QSG-7701 and primary mouse hepatocytes. The survival rates of all cells are shown in Figure 2A. Although the inhibition was not significantly different at $24 \mathrm{~h}$ or $48 \mathrm{~h}$ after baicalin treatment in HCC cell lines, primary mouse hepatocytes were more sensitive to baicalin at $48 \mathrm{~h}$ after treatment $(\mathrm{P}<0.05)$. In order to reduce the toxicity of baicalin on normal hepatocytes, $24 \mathrm{~h}$ after baicalin treatment was chosen in the following mechanism researches. This conclusion will be further corroborated in Figure 3. $\mathrm{IC}_{50}$ values of HCC cells was much higher than normal hepatocytes after $24 \mathrm{~h}$ while no statistical differences in $\mathrm{IC}_{50}$ values between HCC cells and normal hepatocytes after 48h (Figure 2C). Observations of differences between HCC cells and normal hepatocytes in $\mathrm{IC}_{50}$ values demonstrated that baicalin was more toxic to cancer cells than normal hepatocytes at $24 \mathrm{~h}$ after baicalin treatment.

\section{Determination of appropriate nsPEFs parameters}

To determine the appropriate parameters of nsPEFs for the combined treatment, a train of nsPEFs with the doses of $30,40,50,60,80$ in pulse number and the strength of $15 \mathrm{kv} / \mathrm{cm}, 25 \mathrm{kv} / \mathrm{cm}, 40 \mathrm{kv} / \mathrm{cm}$ in electric fields were applied. The reason why we selected 30-80 in pulse number was the generator was set to generate 10 pulses as a group in order to stabilize the wave form and reduce pulse stretching. As shown in Figure 2B, the number of pulses was chosen at 40 pulses because the cell viability after nsPEFs treatment with $40 \mathrm{kv} / \mathrm{cm}$ at more than 40 pulses in HCC-LM3 cell line was too low (about 10\%) and that with $25 \mathrm{kv} / \mathrm{cm}$ and $40 \mathrm{kv} / \mathrm{cm}$ at 30 pulses in QSG-7701 cell line had no statistical difference. Interestingly, although nsPEFs non-selectively killed cells, the tolerance of different cells to nsPEFs was in variety. HCC cell line MHCC-97H seemed to be more susceptible to nsPEFs, while other cells exhibited no apparent difference, which indicated different cell structure might correlate with the different susceptibility to nsPEFs. 

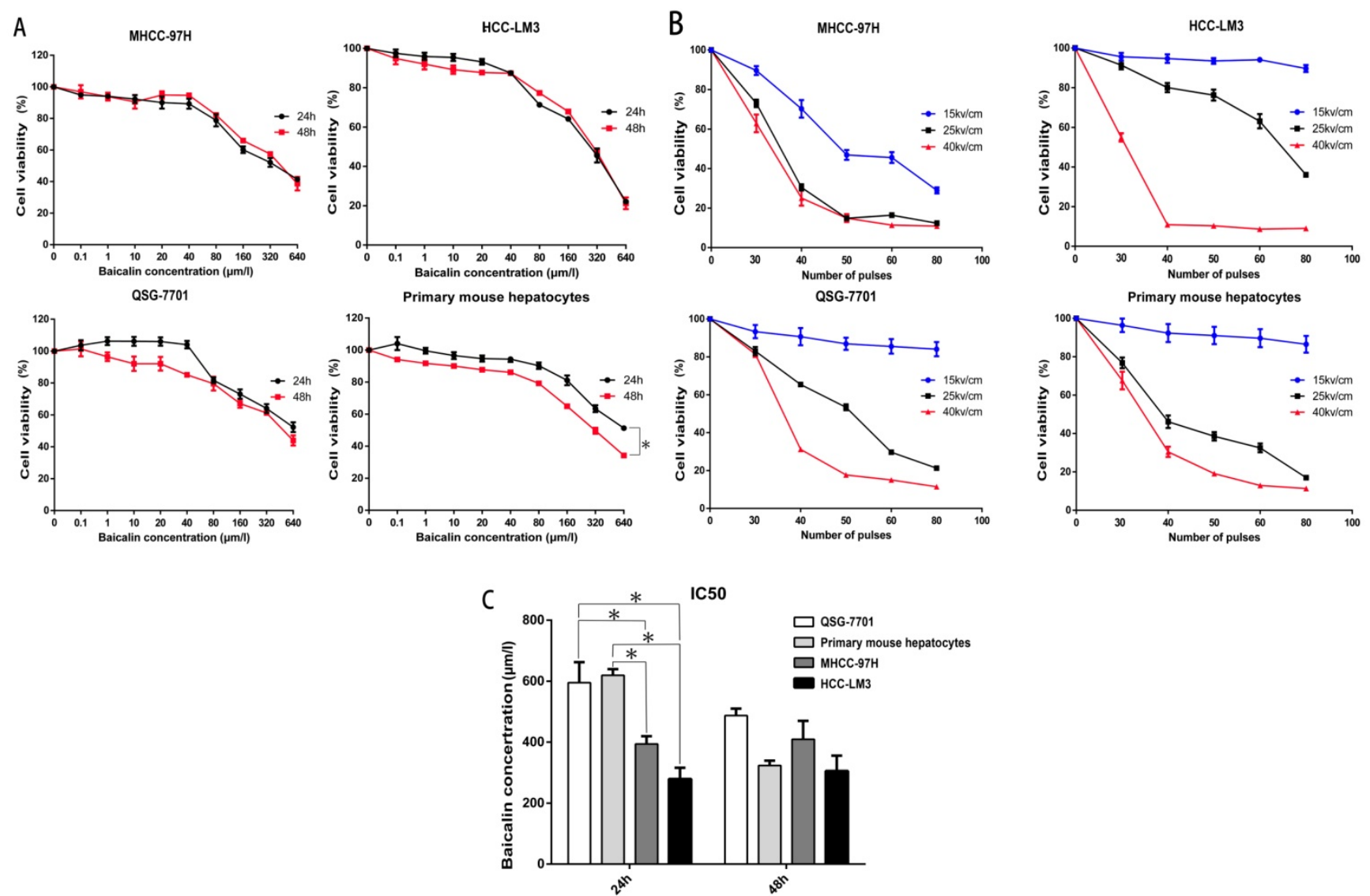

Figure 2: Effect of Baicalin and nsPEFs on cell viability of HCC cell lines and normal liver cells. Different cells were treated with baicalin of indicated concentration $(A, C)$ for $24 \mathrm{~h}$ or $48 \mathrm{~h}$ and nsPEFs with indicated parameters $(B)$ for $24 \mathrm{~h}$. Viabilities were assessed by CCK-8. IC50, half maximal inhibitory concentration. $* \mathrm{P}<0.05$

\section{Combined treatment of nsPEFs and baicalin synergistically inhibited HCC cells whereas protected normal hepatocytes}

After the treatment of $40 \mathrm{P}, 25 \mathrm{kv} / \mathrm{cm}$ nsPEFs combined with a train of baicalin with the concentration of $0-80 \mu \mathrm{M}$ on all cells within $24 \mathrm{~h}$ or $48 \mathrm{~h}$, it appeared that the concentration of $0.625 \mu \mathrm{M}$ was more appropriate for baicalin to alleviate the injury of nsPEFs on normal hepatocytes and enhance the suppression of nsPEFs on HCC cells simultaneously (Figure S1). To further explore the synergistic function of combined application of nsPEFs and baicalin, all cells were treated with $0.625 \mu \mathrm{M}$ baicalin and nsPEFs with 40 pulses and $15,25,40 \mathrm{kv} / \mathrm{cm}$ electric field strength for $24 \mathrm{~h}$ and $48 \mathrm{~h}$. In this regarding experiment, effect difference (ED) value is assessed according to the relative cell viability of the group with nsPEFs treatment alone to that of the group with combined treatment, and the positive or negative value represented synergistic lethal or protective effects, respectively. Figure 3 shows that treatment with $0.625 \mu \mathrm{M}$ baicalin alone had no impact on cell viability within both cancer cells and hepatocytes. However, combined treatment of this relative low concentration of baicalin and nsPEFs with 40 pulses,
$25 \mathrm{kv} / \mathrm{cm}$ caused striking inhibition on both MHCC-97H and HCC-LM3 cell lines compared with the treatment of nsPEFs alone, with the ED value of $20.75,18.51$ at $24 \mathrm{~h}$, respectively and $13.73,15.45$ at $48 \mathrm{~h}$, respectively (Figure 3A-B). Of interest, combined treatment of $0.625 \mu \mathrm{M}$ baicalin and nsPEFs with 40 pulses, a train of $15-40 \mathrm{kv} / \mathrm{cm}$ field strength for $24 \mathrm{~h}$ and $48 \mathrm{~h}$ lead to negative ED values within QSG-7701 cell line and primary mouse hepatocytes (Figure 3C-D). The absolute value of $\mathrm{ED}$ on cell line MHCC-97H, HCC-LM3, QSG-7701 and primary mouse hepatocytes at $24 \mathrm{~h}$ were higher than that at $48 \mathrm{~h}$, which indicated that the synergism of combined treatment at $24 \mathrm{~h}$ was more powerful than $48 \mathrm{~h}$ and further confirmed the conclusion above that $24 \mathrm{~h}$ was the more appropriate time point. These observations suggested completely different lethal mechanisms caused by nsPEFs between HCC cells and normal hepatocytes.

\section{NsPEFs was able to trigger distinct cell death modes}

Since the ED value of $15 \mathrm{kv} / \mathrm{cm}$ and $40 \mathrm{kv} / \mathrm{cm}$ on all cells were too small and had no statistical difference, the appropriate electric field of nsPEFs was $25 \mathrm{kv} / \mathrm{cm}$. In summary, the right parameter of 
combined treatment in the following mechanism experiments was 40 pulses, $25 \mathrm{kv} / \mathrm{cm}$ for nsPEFs and $0.625 \mu \mathrm{M}$ for baicalin within $24 \mathrm{~h}$. To explore the underlying mechanisms of lethal and protective effects, FCM was applied to evaluate the effect of treatment of nsPEFs and/or baicalin on cell death. As shown in Figure 4A-H, the cell death (total number of necrosis and apoptosis) triggered by nsPEFs was enhanced by low concentration of baicalin within MHCC-97H and HCC-LM3 cell lines whilst suppressed within QSG-7701 cell line and primary mouse hepatocytes. In addition, the contribution of combined treatment to ratio of necrotic cells to apoptotic cells was facilitated within MHCC-97H and HCC-LM3 cell lines whereas nearly stable within QSG-7701 cell line and primary mouse hepatocytes (Figure 4I-J), reflecting that there were two different death modes between HCC cells and normal hepatocytes.

In order to confirm the potential distinct cell death modes within HCC and normal liver cells, the protein levels of Poly (ADP-ribose) (PAR) and cleaved PAR polymerase-1 (cleaved PARP-1), which indicated necrosis and apoptosis, respectively, were examined by western-blot assay. PAR is polymerized by PARP-1, a downstream target of activated caspase 3, within the nucleus $[30,31,32]$. In accordance, PAR amount was significantly increased within MHCC-97H and HCC-LM3 cell lines while hardly detected within QSG-7701 cell line and primary mouse hepatocytes after treatment of nsPEFs or combined treatment of nsPEFs and baicalin (Figure $4 \mathrm{~K})$. Moreover, the protein expression of cleaved PARP-1 was hardly examined within MHCC-97H and HCC-LM3 cell lines while remarkably elevated within QSG-7701 cell line and primary mouse hepatocytes after treatment of nsPEFs or combined treatment of nsPEFs and baicalin. Besides, the protein expression of PAR within HCC cells and cleaved PARP-1 within normal hepatocytes were higher and lower, respectively, after combined treatment than nsPEFs treatment alone. Together, above observations mirrored that baicalin treatment was capable of increasing the number of necrotic cells within MHCC-97H and HCC-LM3 cell lines whereas reducing the number of apoptotic cells within QSG-7701 cell line and primary mouse hepatocytes, under the context of nsPEFs treatment. These findings further suggested that, after the treatment of nsPEFs, HCC cell lines and normal hepatocytes exploited two distinct cell death modes, necrosis and apoptosis, respectively, which could be promoted and inhibited by baicalin, respectively.
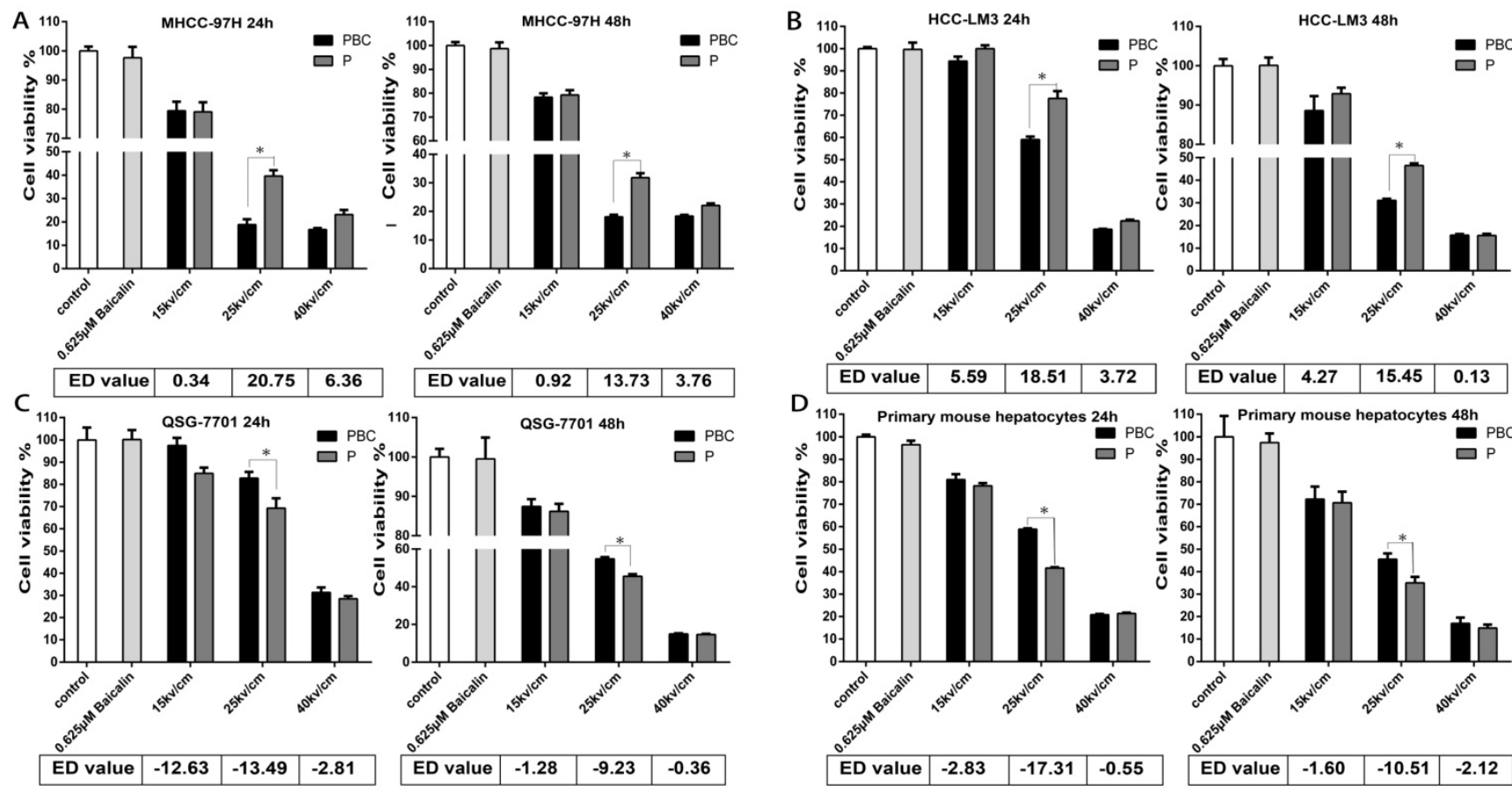

Figure 3: Synergic effect of treatment of nsPEFs and/or baicalin with low concentration on cell viability. Treatment of baicalin with concentration of $0.625 \mu \mathrm{M}$ was little harmful to HCC and normal liver cells. HCC cell lines MHCC-97H (A), HCC-LM3 (B) and normal hepatocyte QSG-7701 (C), primary mouse hepatocytes (D) were treated by baicalin with low concentration $0.625 \mu \mathrm{M}$ and/ or nsPEFs with parameter of $40 \mathrm{P}, 15,25,40 \mathrm{kv} / \mathrm{cm}$ for $24 \mathrm{~h}$ or $48 \mathrm{~h}$, followed by assessment of cell viability. ED value, effect difference value. The ED value were exhibited as mean value of triplicate independent experiments. P, nsPEFs treatment; PBC, combined treatment of nsPEFs and baicalin. $* \mathrm{P}<0.05$ 

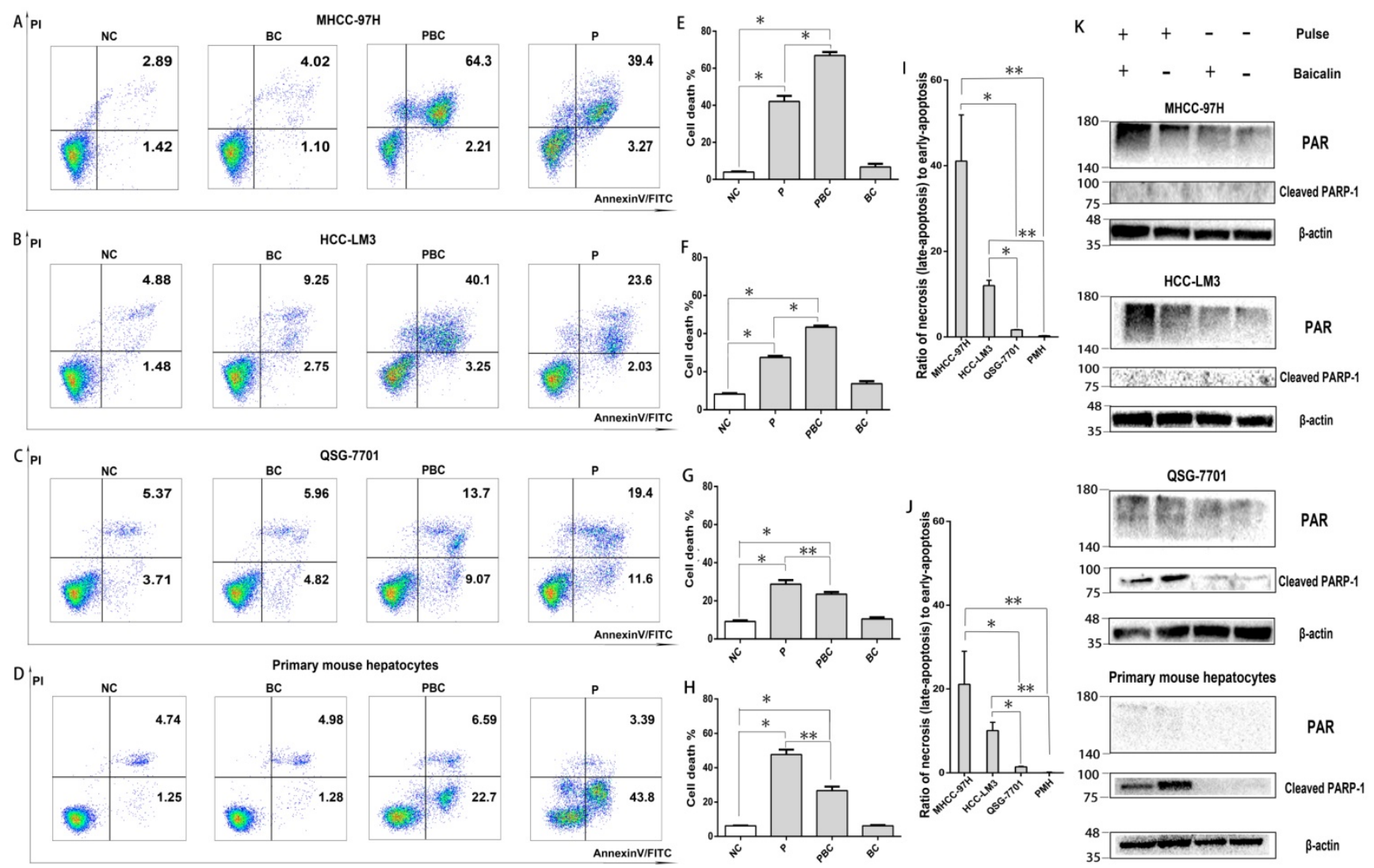

Figure 4: Baicalin enhanced cell necrosis but inhibited apoptosis under nsPEFs treatment. Cell death of MHCC-97H, HCC-LM3, QSG-7701 cell lines and primary mouse hepatocytes after treatment of baicalin and/ or nsPEFs for 24h was evaluated by flow cytometry (A-D). The percentage of cell death (E-H) or ratio of necrosis (or late apoptosis) to early apoptosis for the group with nsPEFs treatment alone $(J)$ and the group with combined treatment (I) were quantified according to flow cytometry results. The amount of cell death markers, PAR and cleaved PARP-1, were examined by western-blot assay (K). PMH: primary mouse hepatocytes; NC, cells without any treatment of nsPEFs or baicalin as negative control; $\mathrm{P}, \mathrm{nsPEFs}$ treatment; $\mathrm{BC}$, baicalin treatment; $\mathrm{PBC}$, combined treatment of nsPEFs and baicalin. $* \mathrm{P}<0.05$, $* * \mathrm{P}<0.01$

\section{Baicalin suppressed nsPEFs-induced mitochondrial transmembrane potential dissipation within normal hepatocytes rather than HCC cell lines}

It has been evident that nsPEFs is able to dissipate MTP and finally result in cell apoptosis [7] while baicalin is protective to injured cells [27]. Therefore, it was reasonable for baicalin to inhibit nsPEFs-mediated cell apoptosis in normal hepatocytes QSG-7701 and primary mouse hepatocytes through reducing MTP dissipation, but not in HCC cell lines MHCC-97H and HCC-LM3. To testify this hypothesis, the alteration of MTP was examined by cationic lipophilic dye JC-1 within HCC cells and normal hepatocytes after nsPEFs treatment or combined treatment of nsPEFs and baicalin. As depicted in Figure 5, the transition of red to green fluorescent signal instantaneously (at $0 \mathrm{~h}$ ) increased in large extent, but decreased in $24 \mathrm{~h}$ within both HCC cells and normal hepatocytes after nsPEFs treatment. Furthermore, application of baicalin could significantly enhance the process of decrease in transition of red to green fluorescent signal within primary mouse hepatocytes and cell line QSG-7701 rather than HCC cell lines MHCC-97H and HCC-LM3 at $24 \mathrm{~h}$. These results demonstrated that the treatment of low concentration of baicalin was able to suppress MTP dissipation triggered by nsPEFs application within normal hepatocytes QSG-7701 and primary mouse hepatocytes but not HCC cell lines MHCC-97H and HCC-LM3.

\section{Baicalin cleared up generation of reactive oxygen species (ROS) after nsPEFs treatment}

Since nsPEFs treatment was capable of triggering accumulation of ROS which could be cleared up by baicalin [33, 34], combined treatment of baicalin probably enabled normal hepatocytes to overcome the oxidative stress caused by nsPEFs and finally to escape from apoptosis. To exactly detect the change of ROS amount after the treatment of nsPEFs or combination of nsPEFs and baicalin, a novel fluorogenic probe for ROS detection which could bind to intracellular DNA as well was utilized. As expected, nsPEFs treatment could significantly promoted ROS production, indicated by dramatic enhancement of green fluorescent signal, which showed the decreasing trend after combined application of nsPEFs and baicalin, especially within 
normal hepatocytes QSG-7701 and primary mouse hepatocytes (Figure 6A-D). These observations were further confirmed by FCM results (Figure 6E-L), reflecting that baicalin was able to clear up intracellular ROS accumulation to some extent, in particular within normal hepatocytes.

\section{Discussion}

NsPEFs is capable of ablating malignancies through various mechanisms, including inducing apoptosis, increasing PM permeability, activating several kinase pathways, etc.[11-17, 19, 35-38] NsPEFs could efficiently ablate tumor lesions through the low-thermal effect [39], but still along with challenges including tumor recurrence or incidences of injury or inflammation within normal tissues $[18,40]$. The current study demonstrated that nsPEFs was able to dramatically kill HCC cells and normal hepatocytes at the same parameters. In addition, a novel strategy of combined treatment with nsPEFs and low concentration of baicalin displayed an inspiring therapeutic outcome that facilitated the suppression of HCC cells whereas attenuated injury of normal hepatocytes through reducing the nsPEFs-triggered MTP dissipation and ROS accumulation.

NsPEFs treatment could cause several cell death modes, consisting of apoptosis, autophagy related apoptosis or necrosis, which were largely dependent on cell types, culture status or nsPEFs parameters [41, 42]. Despite that, few evidences clarify the difference of cell death modes implemented by different cell lines under the treatment of nsPEFs with the same parameter [43]. Here, two distinct cell death modes were uncovered within HCC cell lines and normal hepatocytes. Both results of FCM and protein amount of death markers by immunoblotting showed that cell death of HCC cell lines and normal hepatocytes mainly relied on necrosis and apoptosis, respectively.
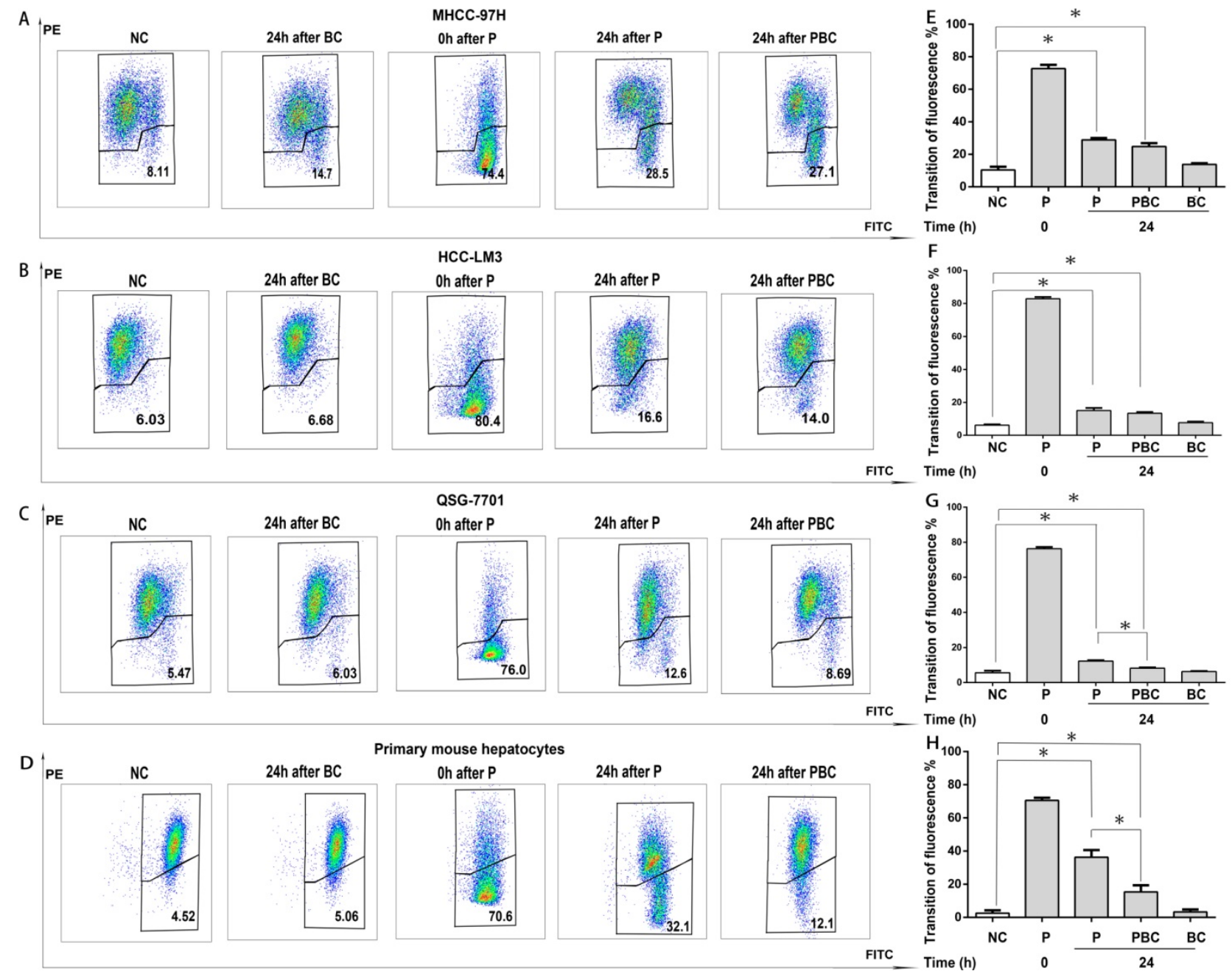

Figure 5: Different functions of baicalin on nsPEFs-mediated dissipation of mitochondria transmembrane potential between normal hepatocytes and HCC cell lines. Both HCC cell lines MHCC-97H, HCC-LM3 and normal hepatocytes QSG-7701, primary mouse hepatocytes were treated by baicalin with concentration $0.625 \mu \mathrm{M}$ and/ or nsPEFs with parameter of $40 \mathrm{P}, 25 \mathrm{kv} / \mathrm{cm}$ for indicated time, and then their mitochondria transmembrane potential was detected using $\mathrm{CC}-1$ assay through FCM. $\mathrm{PE}$ and FITC represented red and green fluorescent signal detected by FCM, respectively (A-D). The quantification of transition of red fluorescent signal to green fluorescent signal $(\mathrm{E}-\mathrm{H})$. NC, cells without any treatment of nsPEFs or baicalin as negative control; P, nsPEFs treatment; BC, baicalin treatment; PBC, combined treatment of nsPEFs and baicalin. $* \mathrm{P}<0.05$ 
Specifically, the characteristics of dead HCC cells were double positive $\mathrm{PI}(+) \mathrm{FITC}(+)$ by $\mathrm{FCM}$ and increase in protein amount of PAR by immunoblot, differing from single positive FITC $(+)$ and increased protein level of cleaved PARP-1 by immunoblot within normal hepatocytes. Similarly, dependence on necrosis of malignant cells after nsPEFs treatment was unveiled in the study of nsPEFs treatment on lymphadenoma cell line U937 [41]. However, another inconsistent evidence revealed that melanoma cancer cells are destructed by nsPEFs through apoptosis [19]. This discrepancy might be caused by the different parameters of diverse nsPEFs generator, for instance, pulse width, electric fields strength, pulse stretching or pulse frequency etc. Furthermore, it remained further confirmation that these distinct death modes also existed in malignant and normal cells originated from other organs.

The dual-function of baicalin on anti-tumor and anti-oxidation $[23,29]$ clued that baicalin might be an ideal pharmacological agent for enhancing ablation effect of tumor and alleviating complications by nsPEFs treatment. Of interest, combined treatment of low concentration of baicalin exhibited the ability to promote the death of HCC cells and reduce the death of normal hepatocytes caused by nsPEFs treatment. Sole application of baicalin with low concentration was quite safe for cell survival (Figure 2-3), differing from killing effects of baicalin with a higher concentration on tumor cells reliable on apoptosis pathway [28, 29]. However, treatment of baicalin with higher concentration would be harmful to the survival of normal hepatocytes, which could possibly be accountable for the priority of cell death pathways over anti-oxidation or cell survival protection pathways. Due to distinct cell death modes induced by nsPEFs, necrosis within HCC cells and apoptosis within normal hepatocytes, difference in loss of plasma membrane integrity would probably allow different intracellular accumulation of agents [41], such as baicalin, and eventually affect the fate of different cells.

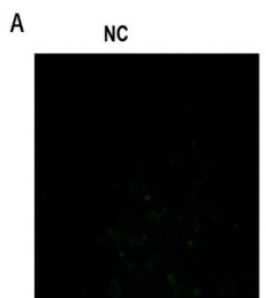

B

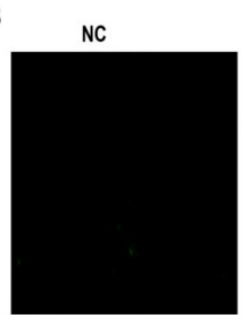

C
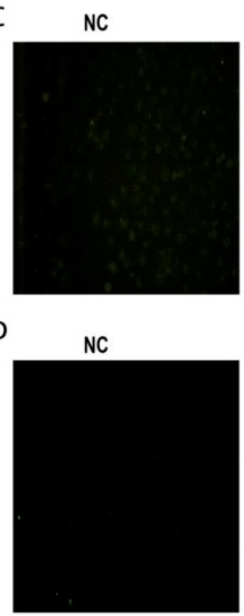

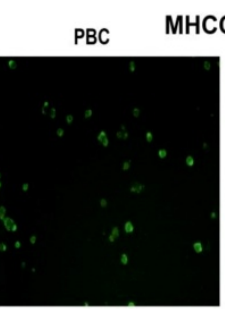

PBC

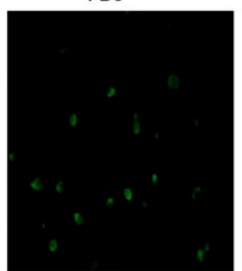

PBC

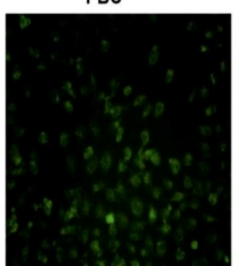

Primary mouse hepatocytes

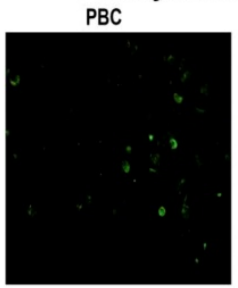

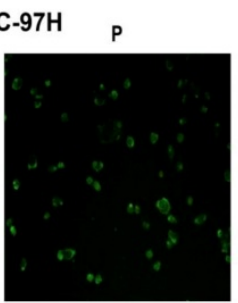

CC-LM3

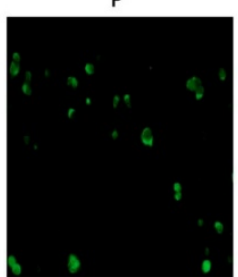

QSG-7701

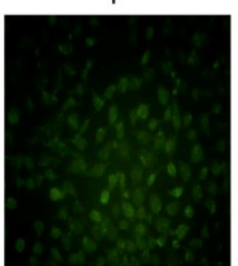

$P$

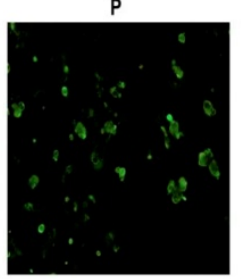

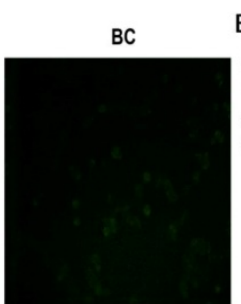

BC

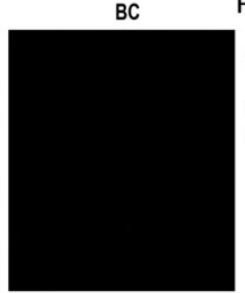

$\mathrm{BC}$

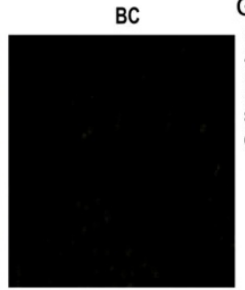

$\mathrm{BC}$

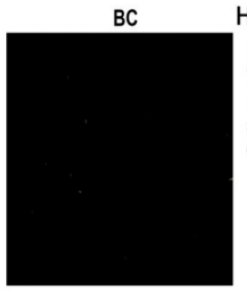

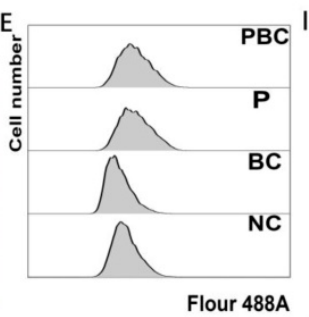

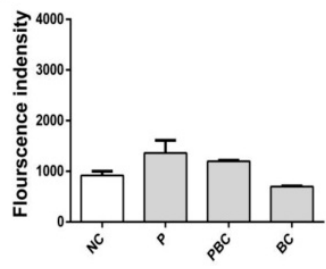

J
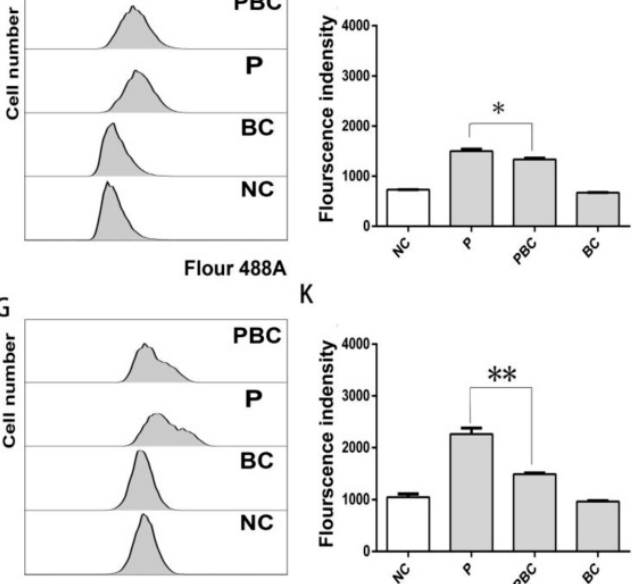

Flour 488A
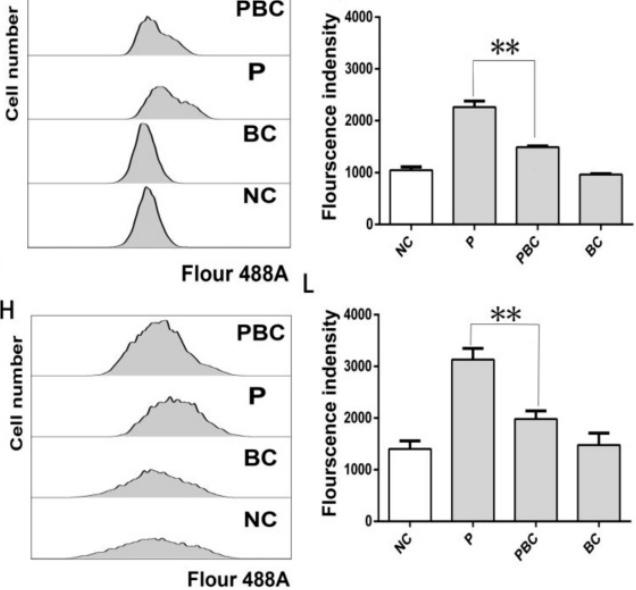

Figure 6: Baicalin cleared up nsPEFs-induced ROS accumulation within normal hepatocyte. HCC cell lines MHCC-97H, HCC-LM3 and normal hepatocyte QSG-7701, primary mouse hepatocytes were treated by baicalin with concentration $0.625 \mu \mathrm{M}$ and/ or nsPEFs with parameter of $40 \mathrm{P}, 25 \mathrm{kv} / \mathrm{cm}$ for indicated time, and then their ROS production was detected by CellROX Green Reagent through fluorescent microscopy (A-D) or FCM (E-H). Transition of red fluorescent signal to green fluorescent signal was the quantified (I-L). NC, cells without any treatment of nsPEFs or baicalin as negative control; P, nsPEFs treatment; BC, baicalin treatment; PBC, combined treatment of nsPEFs and baicalin. $* \mathrm{P}<0.05 * * \mathrm{P}<0.01$ 


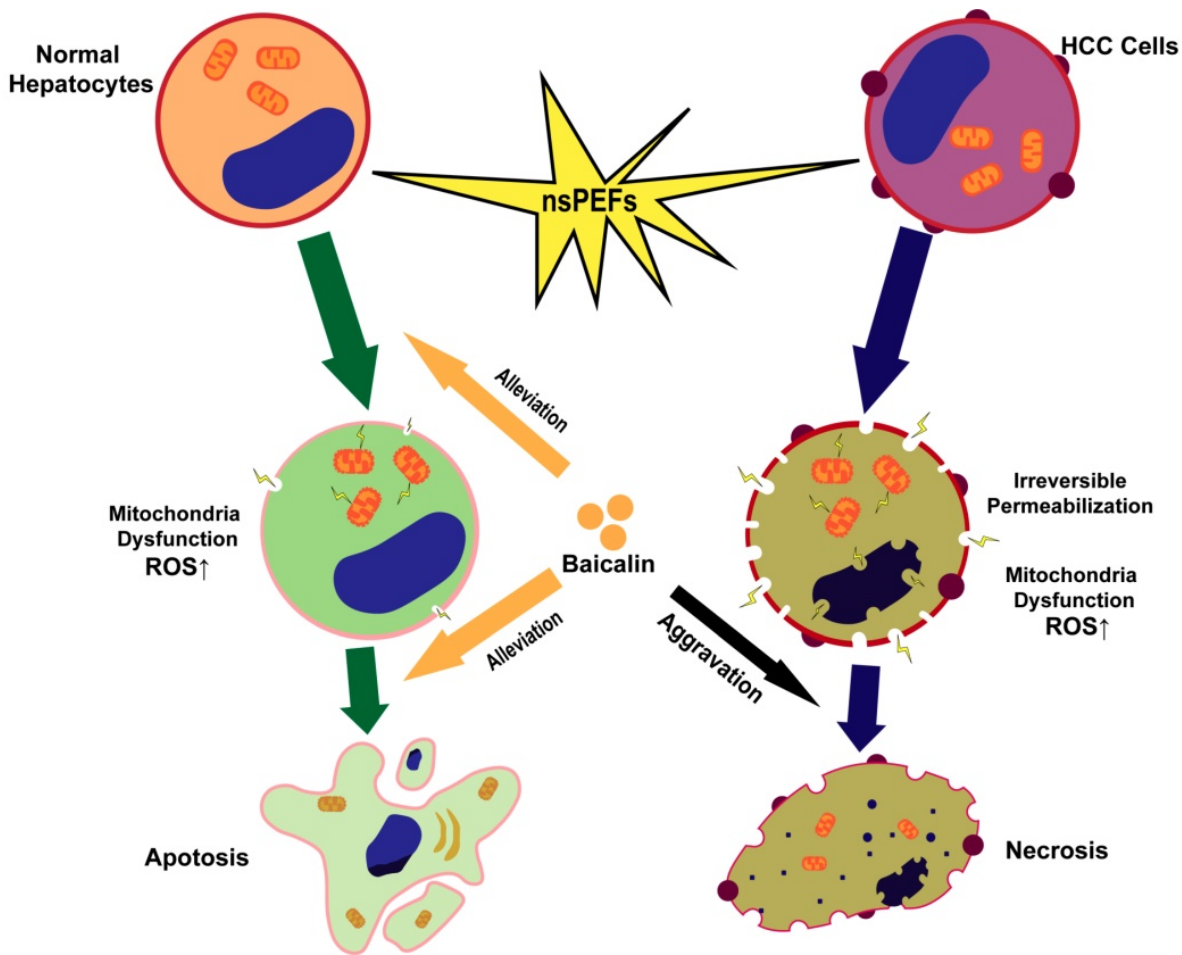

Figure 7: Schematic illustration of mechanism that nsPEFs-triggered cell death was suppressed within normal hepatocytes whilst facilitated within HCC cells by baicalin.

Intracellular ROS accumulation and MTP dissipation, two typical features of cells after treatment of nsPEFs, play key role in cell death [35]. This was substantiated by our findings including spontaneous ROS production with a large amount and MTP dissipation with different degrees within both HCC and normal liver cells. Given roles in anti-oxidant stress $[22,23]$ and the close correlation with ROS, MTP and apoptosis [44-46], baicalin possesses the potential to clear up nsPEFs-induced intracellular ROS accumulation and stabilize MTP and ultimately overcomes cell death of normal hepatocytes under nsPEFs treatment. As expected, our results demonstrated that combined usage of baicalin with low concentration suppressed the dissipation of MTP and reduced the accumulation of ROS within normal hepatocytes QSG-7701 and primary mouse hepatocytes after the application of nsPEFs, in agreement with the effect of baicalin on the reverse of ultraviolet radiation-induced oxidative damage [27]. However, treatment of baicalin with low concentration could induce a descending trend of nsPEFs-induced ROS generation but not MTP recovery within HCC cell line HCC-LM3, supporting that baicalin could clear up ROS non-selectively [47]. Additionally, MTP dissipation within necrotic cells [48], resulting in a decrease of ATP and dysfunction of mitochondria, was tougher to overcome [49]. The present observations confirmed that nsPEFs-induced MTP dissipation within necrotic HCC cells was more difficult to recover despite the reduction of ROS accumulation to certain extend within HCC cells by low concentration of baicalin. This could explain that baicalin could not attenuate nsPEFs-induced cell death of HCC cell lines.

In conclusion, HCC cells and normal hepatocytes are killed by nsPEFs mainly through necrosis and apoptosis, respectively. NsPEFs-induced cell deaths of HCC and normal liver cells can be promoted and attenuated by baicalin with low concentration, respectively, and baicalin prevents normal hepatocytes from damage by nsPEFs largely through clearing up ROS production and stabilizing MTP (Figure 7). Our findings provide an advantageous therapy for HCC that combined treatment of nsPEFs and baicalin could improve the tumor-ablation effect as well as reduce complications of the clinical application of nsPEFs.

\section{Abbreviations}

HCC: hepatocellular carcinoma; nsPEFs: nanosecond pulsed electric fields; ROS: reactive oxygen species; MTP: mitochondrial transmembrane potential; ATP: adenosine triphosphate; PM: plasma membrane; TACE: trans arterial chemoembolization; RFA: radiofrequency ablation; PEI: percutaneous ethanol injection; CCK-8: cell counting kit 8; FCM: flow cytometry; PAR: poly (ADP-ribose).

\section{Supplementary Material}

Supplementary figure.

http://www.medsci.org/v16p1271s1.pdf 


\section{Acknowledgments}

This research was supported by Innovative Research Groups of National Natural Science Foundation of China (81721091), Major Program of National Natural Science Foundation of China (91542205), National Science and Technology Foundation of China (81572954), National S\&T Major Project of China (2018ZX10301201-006-001, 2017ZX10203205), Zhejiang International Science and Technology Cooperation Project (2016C04003) and Zhejiang Science and Technology Project of traditional Chinese Medicine (2018ZB072).

\section{Competing Interests}

The authors have declared that no competing interest exists.

\section{References}

1. Bray F, Ferlay J, Soerjomataram I, Siegel RL, Torre LA, Jemal A. Global cancer statistics 2018: GLOBOCAN estimates of incidence and mortality worldwide for 36 cancers in 185 countries. CA Cancer J Clin. 2018; 68(6):394-424

2. Bleicher RJ, Allegra DP, Nora DT, Wood TF, Foshag LJ, Bilchik AJ. Radiofrequency Ablation in 447 Complex Unresectable Liver Tumors: Lessons Learned. Annals of Surgical Oncology. 2003; 10(1):52-58.

3. Breton M, Mir LM. Microsecond and nanosecond electric pulses in cancer treatments. Bioelectromagnetics. 2012; 33(2):106-123.

4. Himoto T, Kurokohchi K, Watanabe S, Masaki T. Recent advances in radiofrequency ablation for the management of hepatocellular carcinoma. Hepat Mon. 2012; 12(10 HCC):e5945.

5. Chen R, Chen X. Nanosecond Pulsed Electric Field (nsPEF) Ablation as an Alternative or Adjunct to Surgery for Treatment of Cancer. Surgery: Current Research. 2013; 01(s12).

6. Karl H. Schoenbach, Stephen J. Beebe, E. Stephen Buescher. Intracellular Effect of Ultrashort Electrical Pulses. Bioelectromagnetics. 2001; 22:440-448.

7. Beebe SJ, Chen YJ, Sain NM, Schoenbach $\mathrm{KH}$, Xiao S. Transient features in nanosecond pulsed electric fields differentially modulate mitochondria and viability. PLoS One. 2012; 7(12):e51349.

8. Vernier PT, Sun Y, Marcu L, Salemi S, Craft CM, Gundersen MA. Calcium bursts induced by nanosecond electric pulses. Biochemical and Biophysical Research Communications. 2003; 310(2):286-295.

9. Scarlett SS, White JA, Blackmore PF, Schoenbach KH, Kolb JF. Regulation of intracellular calcium concentration by nanosecond pulsed electric fields. Biochim Biophys Acta. 2009; 1788(5):1168-1175.

10. Batista Napotnik T, Rebersek M, Vernier PT, Mali B, Miklavcic D. Effects of high voltage nanosecond electric pulses on eukaryotic cells (in vitro): A systematic review. Bioelectrochemistry. 2016; 110:1-12.

11. Beebe SJ, White J, Blackmore PF, Deng YP, Somers K, Schoenbach KH. Diverse Effects of Nanosecond Pulsed Electric Fields on Cells and Tissues. DNA And Cell Biology. 2003; 22(12):785-796

12. Morotomi-Yano K, Oyadomari S, Akiyama H, Yano K. Nanosecond pulsed electric fields act as a novel cellular stress that induces translational suppression accompanied by eIF2alpha phosphorylation and 4E-BP1 dephosphorylation. Exp Cell Res. 2012; 318(14):1733-1744.

13. Hall EH, Schoenbach KH, Beebe SJ. Nanosecond pulsed electric fields induce apoptosis in p53-wildtype and p53-null HCT116 colon carcinoma cells. Apoptosis. 2007; 12(9):1721-1731.

14. Beebe SJ, Sain NM, Ren W. Induction of Cell Death Mechanisms and Apoptosis by Nanosecond Pulsed Electric Fields (nsPEFs). Cells. 2013; 2(1):136-162.

15. Morotomi-Yano K, Akiyama H, Yano K. Nanosecond pulsed electric fields activate AMP-activated protein kinase: implications for calcium-mediated activation of cellular signaling. Biochem Biophys Res Commun. 2012; 428(3):371-375

16. Morotomi-Yano K, Akiyama H, Yano K. Nanosecond pulsed electric fields activate MAPK pathways in human cells. Arch Biochem Biophys. 2011; 515(1-2):99-106.

17. Ren Z, Chen X, Cui G, Yin S, Chen L, Jiang J, Hu Z, Xie H, Zheng S, Zhou L. Nanosecond pulsed electric field inhibits cancer growth followed by alteration in expressions of NF-kappaB and Wnt/beta-catenin signaling molecules. PLoS One. 2013; 8(9):e74322.

18. Chen X, .Zhuang J, Kolb JF, Schoenbach KH, Beebe SJ. Long Term Survival of Mice With Hepatocellular Carcinoma after Pulse Power Ablation with Nanosecond Pulsed Electric Fields. Technology in Cancer Research and Treatment. 2012; 11(1):83-93.
19. Chen X, Kolb JF, Swanson RJ, Schoenbach KH, Beebe SJ. Apoptosis initiation and angiogenesis inhibition: melanoma targets for nanosecond pulsed electric fields. Pigment Cell Melanoma Res. 2010; 23(4):554-563.

20. Stacey M, Osgood C, Kalluri BS, Cao W, Elsayed-Ali H, Abdel-Fattah T. Nanosecond pulse electrical fields used in conjunction with multi-wall carbon nanotubes as a potential tumor treatment. Biomed Mater. 2011; 6(1):011002.

21. Yin D, Yang WG, Weissberg J, Goff CB, Chen W, Kuwayama Y, Leiter A, Xing $\mathrm{H}$, Meixel A, Gaut D et al. Cutaneous papilloma and squamous cell carcinoma therapy utilizing nanosecond pulsed electric fields (nsPEF). PLoS One. 2012; 7(8):e43891.

22. Cao Y, Mao X, Sun C, Zheng P, Gao J, Wang X, Min D, Sun H, Xie N, Cai J. Baicalin attenuates global cerebral ischemia/reperfusion injury in gerbils via anti-oxidative and anti-apoptotic pathways. Brain Res Bull. 2011; 85(6):396-402.

23. Gao Z, Huang $\mathrm{K}, \mathrm{Xu} \mathrm{H}$. Protective effects of flavonoids in the roots of Scutellaria baicalensis Georgi against hydrogen peroxide-induced oxidative stress in HS-SY5Y cells. Pharmacol Res. 2001; 43(2):173-178.

24. Shen Y-C, Chiou W-F, Chou Y-C, Chen C-F. Mechanisms in mediating the anti-inflammatory effects of baicalin and baicalein in human leukocytes. European Journal of Pharmacology. 2003; 465(1-2):171-181.

25. Yoshiharu Motoo, Norio Sawabu. Antitumor effects of saikosaponins, baicalin and baicalein on human hepatoma cell lines. Cancer Letters. 1994; 86: 91-95.

26. Chen $\mathrm{CH}$, Huang LL, Huang CC, Lin CC, Lee Y, Lu FJ. Baicalein, a novel apoptotic agent for hepatoma cell lines: a potential medicine for hepatoma. Nutr Cancer. 2000; 38(2):287-295

27. Zhou BR, Yin HB, Xu Y, Wu D, Zhang ZH, Yin ZQ, Permatasari F, Luo D. Baicalin protects human skin fibroblasts from ultraviolet A radiation-induced oxidative damage and apoptosis. Free Radic Res. 2012; 46(12):1458-1471.

28. Chiu YW, Lin TH, Huang WS, Teng CY, Liou YS, Kuo WH, Lin WL, Huang $\mathrm{HI}$, Tung JN, Huang CY et al. Baicalein inhibits the migration and invasive properties of human hepatoma cells. Toxicol Appl Pharmacol. 2011; 255(3):316-326.

29. Yu Y, Pei MY, Li L. Baicalin induces apoptosis in hepatic cancer cells in vitro and suppresses tumor growth in vivo. Int J Clin Exp Med. 2015; 8(6):8958-8967.

30. Luo $X$, Kraus WL. On PAR with PARP: cellular stress signaling through poly(ADP-ribose) and PARP-1. Genes Dev. 2012; 26(5):417-432.

31. Virag L, Robaszkiewicz A, Rodriguez-Vargas JM, Oliver FJ. Poly(ADP-ribose) signaling in cell death. Mol Aspects Med. 2013; 34(6):1153-1167.

32. Morotomi-Yano K, Akiyama H, Yano K. Different involvement of extracellular calcium in two modes of cell death induced by nanosecond pulsed electric fields. Arch Biochem Biophys. 2014; 555-556:47-54.

33. Nuccitelli R, Lui K, Kreis M, Athos B, Nuccitelli P. Nanosecond pulsed electric field stimulation of reactive oxygen species in human pancreatic cancer cells is $\mathrm{Ca}(2+)$-dependent. Biochem Biophys Res Commun. 2013; 435(4):580-585.

34. Pakhomova ON, Khorokhorina VA, Bowman AM, Rodaite-Riseviciene $R$ Saulis G, Xiao S, Pakhomov AG. Oxidative effects of nanosecond pulsed electric field exposure in cells and cell-free media. Arch Biochem Biophys. 2012; 527(1):55-64.

35. Yin S, Chen X, Hu C, Zhang X, Hu Z, Yu J, Feng X, Jiang K, Ye S, Shen K et al. Nanosecond pulsed electric field (nsPEF) treatment for hepatocellular carcinoma: a novel locoregional ablation decreasing lung metastasis. Cancer Lett. 2014; 346(2):285-291.

36. Wu S, Guo JS, Wei WD, Zhang J, Fang J, Beebe SJ. Enhanced breast cancer therapy with nsPEFs and low concentrations of gemcitabine. Cancer Cell International. 2014; 14(98).

37. Qi W, Guo J, Wu S, Su B, Zhang L, Pan J, Zhang J. Synergistic effect of nanosecond pulsed electric field combined with low-dose of pingyangmycin on salivary adenoid cystic carcinoma. Oncol Rep. 2014; 31(5):2220-2228.

38. Wang J, Guo J, Wu S, Feng H, Sun S, Pan J, Zhang J, Beebe SJ. Synergistic effects of nanosecond pulsed electric fields combined with low concentration of gemcitabine on human oral squamous cell carcinoma in vitro. PLoS One. 2012; 7(8):e43213.

39. Ng KK, Poon RT, Lam CM, Yuen J, Tso WK, Fan ST. Efficacy and safety of radiofrequency ablation for perivascular hepatocellular carcinoma without hepatic inflow occlusion. Br J Surg. 2006; 93(4):440-447.

40. Xie F, Varghese F, Pakhomov AG, Semenov I, Xiao S, Philpott J, Zemlin C. Ablation of Myocardial Tissue With Nanosecond Pulsed Electric Fields. PLoS One. 2015; 10(12):e0144833.

41. Pakhomova ON, Gregory BW, Semenov I, Pakhomov AG. Two modes of cell death caused by exposure to nanosecond pulsed electric field. PLoS One. 2013; 8(7):e70278

42. Ullery JC, Tarango M, Roth CC, Ibey BL. Activation of autophagy in response to nanosecond pulsed electric field exposure. Biochem Biophys Res Commun. 2015; 458(2):411-417

43. Gianulis EC, Labib C, Saulis G, Novickij V, Pakhomova ON, Pakhomov AG. Selective susceptibility to nanosecond pulsed electric field (nsPEF) across different human cell types. Cell Mol Life Sci. 2017; 74(9):1741-1754.

44. Yang Y, Karakhanova S, Hartwig W, D'Haese JG, Philippov PP, Werner J, Bazhin AV. Mitochondria and Mitochondrial ROS in Cancer: Novel Targets for Anticancer Therapy. J Cell Physiol. 2016; 231(12):2570-2581.

45. Zhou R, Yazdi AS, Menu P, Tschopp J. A role for mitochondria in NLRP3 inflammasome activation. Nature. 2011; 469(7329):221-5.

46. La Russa D, Brunelli E, Pellegrino D. Oxidative imbalance and kidney damage in spontaneously hypertensive rats: activation of extrinsic apoptotic pathways. Clin Sci (Lond). 2017; 131(13):1419-1428. 
47. Srinivas NR: Baicalin, an emerging multi-therapeutic agent. Pharmacodynamics, pharmacokinetics, and considerations from drug development perspectives. Xenobiotica. 2010; 40(5):357-367.

48. Sunaga D, Tanno M, Kuno A, Ishikawa S, Ogasawara M, Yano T, Miki T, Miura T. Accelerated recovery of mitochondrial membrane potential by GSK-3beta inactivation affords cardiomyocytes protection from oxidant-induced necrosis. PLoS One. 2014; 9(11):e112529.

49. Eguchi Y, Shimizu S, Tsujimoto Y. Intracellular ATP levels determine cell death fate by apoptosis or necrosis. Cancer Research. 1997; 57:1835-1840. 